\title{
Exploring the Possibility of Virtual Reality in Nursing Skills Education: A Preliminary Study Using a First-Person Video
}

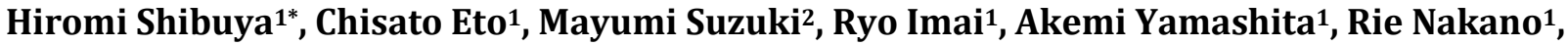 Saori Kawanabe', Motomi Yokota ${ }^{1}$, Satoshi Shibuya ${ }^{3}$ (])}

\author{
${ }^{1}$ Department of Nursing, Faculty of Health Science Technology, Bunkyo Gakuin University, Tokyo, Japan \\ ${ }^{2}$ Nippon Medical School Hospital, Tokyo, Japan \\ ${ }^{3}$ Department of Integrative Physiology, School of Medicine, Kyorin University, Tokyo, Japan \\ Email: *hshibuya@bgu.ac.jp
}

How to cite this paper: Shibuya, H., Eto, C., Suzuki, M., Imai, R., Yamashita, A., Nakano, R., Kawanabe, S., Yokota, M. and Shibuya, S. (2019) Exploring the Possibility of Virtual Reality in Nursing Skills Education: A Preliminary Study Using a First-Person Video. Open Journal of Nursing, 9, 163-172. https://doi.org/10.4236/ojn.2019.92015

Received: January 18, 2019

Accepted: February 18, 2019

Published: February 21, 2019

Copyright $\odot 2019$ by author(s) and Scientific Research Publishing Inc. This work is licensed under the Creative Commons Attribution International License (CC BY 4.0).

http://creativecommons.org/licenses/by/4.0/

(C) (i) Open Access

\begin{abstract}
Background: While demonstration videos from a third-person perspective have traditionally been used as teaching materials for nursing skills, first-person videos allow observers to experience how nursing educators move and what they see. In this pilot study, a first-person demonstration video for tracheostomy suctioning skills was shown to participants via a virtual reality (VR) head-mounted display (HMD), which we referred to as a VR teaching material (VR-TM). In an intervention experiment, we investigated the effects of the VR-TM on participants' performance on the skill assessment test. Additionally, usability of the VR-TM was evaluated by questionnaire ratings. Methods: Thirty-six participants (nursing students) were assigned to three groups in the experiment: 1) VR group: the participants that viewed the VR-TM, 2) TR group: the participants that viewed a traditional demonstration video from a third-person perspective, and 3) NO group: the participants that received no intervention. Score changes in the skill assessments of participants before the intervention (pre-test) and after the intervention (post-test) were analyzed. After the experiment, all participants completed a faculty-designed questionnaire about the VR-TM usability. Results: The questionnaire ratings indicated that the participants evaluated the VR-TM favorably in terms of skill understanding and motivation to practice the skill. However, one-third of participants reported mild discomfort during the VR-TM. In regards to score changes in the skill assessment test, no significant difference between the VR and TR groups was identified, though the two groups produced greater score changes compared to the NO group. Conclusion: The combination of first-person video and the HMD might be promising as a
\end{abstract}


teaching material for nursing skills, though further improvements are necessary.

\section{Keywords}

Virtual Reality, Nursing Education, Tracheostomy Suctioning, Demonstration Video

\section{Introduction}

While nursing students must learn many medical procedures as nursing skills, they can only practice them in a school's laboratory where there are patient simulators and medical instruments (i.e. simulation-based training (SBT)). After passing achievement tests for the skills, the students must remember them and perform the skills on patients. Therefore, teaching materials like demonstration videos are important tools that support SBT and help students retain learned skills. Due to technological limitations, demonstration videos traditionally utilized as teaching materials for nursing skills have been limited to a third-person perspective. However, virtual reality (VR) technology now enables demonstrators to create first-person videos that lead observers to experience what nursing educators see and how they move their hands [1].

In various fields of education, the introduction of VR has grown in recent years. VR technology can provide users a simulated experience with a first-person perspective via various sensory stimulations [2]. Given that experience-based knowledge, not mere book knowledge, is needed in nursing education; VR could be a promising educational tool. While the application of VR in nursing education is a recent innovation [3], a Japanese private company recently developed a VR teaching tool to help medical professional empathize with patients with dementia. This allows users to have an immersive experience of the disease (i.e. hallucination and memory impairment). Similarly, in the University of New England in Australia, an educational project using immersive VR software aims to teach empathy regarding the aging process to health profession students by leading the learners through a simulated aging experience (e.g. macular degeneration and hearing loss) [4].

VR is also being used for nursing skills training. Boise State University in the USA has developed a computer graphics (CG)-based VR system with haptic feedback for practicing urinary catheterization [5]. An evaluation of this system found that the participants who experienced it evaluated it favorably, spent more time practicing, and finished more procedures in one hour compared to the control participants who practiced the skill in the traditional way. Though this type of VR system is advanced, it seems to be difficult to apply to the nursing education field because the development of CG-based VR systems is costly and requires in-depth knowledge of computer science and engineering. 
In this preliminary study, a first-person demonstration video for tracheostomy suctioning skills was developed and shown to participants via an immersive VR head-mounted display (HMD). We examined the usefulness of this VR demonstration video through an intervention experiment and a faculty-designed questionnaire.

\section{Methods}

\subsection{Participants}

Thirty-six third- and fourth-year students at Bunkyo Gakuin University (8 men and 28 women; aged 21 to 23 years) participated in this study. All participants had normal or corrected-to-normal vision. This study was approved by the institutional human review board at Bunkyo Gakuin University (approval No. 2018-0008), and all participants provided written informed consent in accordance with institutional guidelines. The sample size was decided using the $\mathrm{G}^{\star}$ Power software for power analysis, version 3.1.9. The alpha value $(\alpha)$, power $(1-\beta)$ and effect size (f) as setting parameters were $0.05,0.7$ and 0.49 , respectively.

\subsection{Video Production}

Based on ethical considerations for the participants, tracheostomy suctioning was chosen as the teaching skill because all participants had learned it and previously passed an achievement test as second-year students. To create video footage from a first-person perspective, a nurse educator wore a wearable camera (Panasonic HX-A500) on her head and demonstrated tracheostomy suctioning with a patient simulator while recording (Figure 1(a)). The raw video footage was edited into a six-minute teaching video using a video editing software (Adobe Premiere Elements, version 15) (Figure 1(b)), which we will refer to as the VR teaching material (VR-TM). In the VR-TM, some narrations were inserted to explain technically important points of tracheostomy suctioning. Participants viewed the VR-TM via the HMD for gaming (Sony PlayStation VR) (Figure 1(c)).

Using a commercial video camera (Sony HDR-CX470) that was fixed beside the patient's bed, we recorded the same educator's demonstration from a third-person perspective. Similar to the VR-TM, the raw video footage was edited into a six-minute teaching video (Figure 1(d)), which we will refer to as the traditional teaching material (TR-TM). Consequently, the contents between the VR-TM and TR-TM were almost identical (e.g. demonstrator, narration and duration of the video footage), except for perspectives (i.e. first-person vs third-person). The TR-TM was shown to some participants via a 15-inch laptop (NEC PC-SN254).

\subsection{Study Design}

A schematic representation of the study design is shown in Figure 2. After the informed consent document had been signed, all participants were given the 
tracheostomy suctioning skill assessment pretest. Subsequently, all participants were pseudo-randomly assigned to one of three groups ( $n=12$ in each group) in an intervention phase (a dashed square in Figure 2): 1) VR group: the participants viewed the VR-TM once, 2) TR group: the participants viewed the TR-TM once, and 3) NO group: the participants rested for $10 \mathrm{~min}$ and received no intervention. After the intervention phase, the skill assessment posttest was given to all participants. After the experiment was completed, both the TR and NO groups also viewed the VR-TM. Finally, all participants completed a faculty-designed questionnaire about the VR-TM usability.

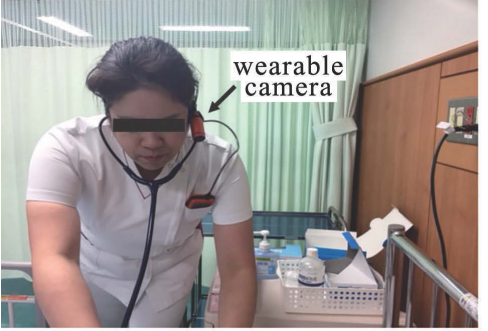

(a)

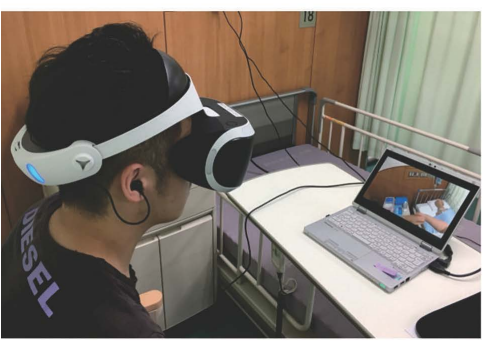

(c)

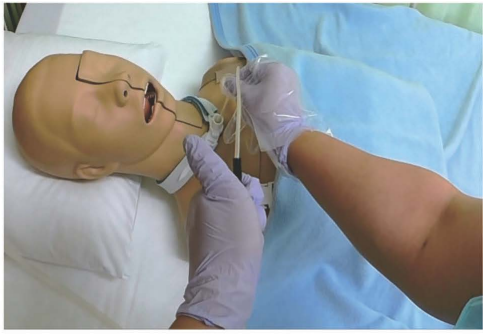

(b)

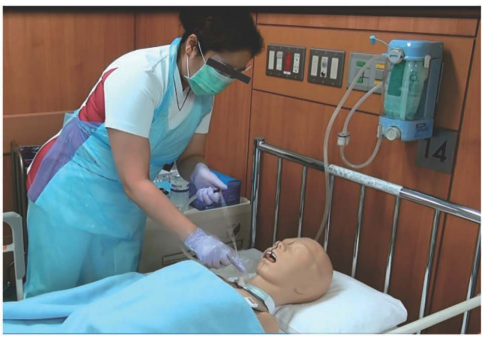

(d)

Figure 1. Recordings of an educator's demonstration using a wearable camera (a). A screenshot of the first-person video (b). A nursing student watching the VR teaching material with a head-mounted display (c). A screenshot of the third-person video (d).

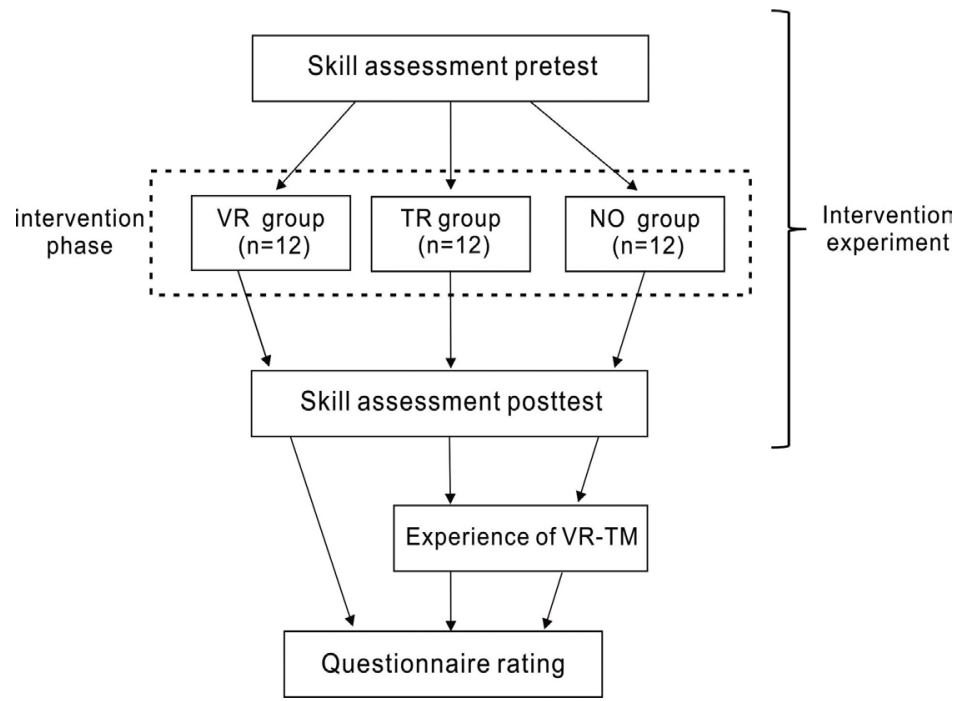

Figure 2. A schematic representation of the study design (VR-TM: virtual reality teaching material). 


\subsection{Skill Assessment Test}

In the skill assessment test, each participant was required to demonstrate tracheostomy suctioning using the patient simulator, which was assessed by two nurses as evaluators (not authors) with a standard 24-item checklist (Table 1). The concordance rate between the two evaluators was greater than $90 \%$, and mean scores between the two were used for data analyses.

\subsection{Questionnaire for VR-TM Usability}

To measure VR-TM usability, the authors made a questionnaire with a 5-point Likert scale, ranging from +2 (agree strongly) to -2 (disagree strongly). This questionnaire consisted of 12 items (Table 2), which were divided into the following five categories according to the statements: understanding of the skill (Q1-3), motivation to practice the skill (Q4-6), immersion to VR (Q7-9), control (Q10-Q11), and side effects (Q12). Q1-9 served as target items to assess usability, whereas Q10-11 served as control items (reversed items) to verify response reliability. Q12 was added to examine side effects of the VR-TM (i.e. VR sickness).

Table 1. The checklist of tracheostomy suctioning skills (0: Unsatisfactory or 1: Satisfactory).
1) Identify patient using name and explain what is about to occur
2) Auscultate bronchial breath sounds
3) Perform hand hygiene before donning personal protective equipment (PPE)
4) Don PPE in the correct order (disposable apron, mask with face shield and disposable gloves)
5) Set suction pressure accurately (between 13 and $20 \mathrm{kPa}$ )
6) Connect the suction catheter to the connecting tubing
7) Remove cap from sterile water and don sterile gloves (dominant hand)
8) Pick up the sterile suction catheter without touching any non-sterile surfaces
9) Pick up the suction catheter (sterile portion) with sterile (dominant) hand
10) Turn on suction machine and aspirate sterile water
11) Explain to patient that you are about to suction tracheostomy
12) Remove artificial nose with the non-sterile (non-dominant) hand
13) Position artificial nose on package of sterile gloves (sterile place)
14) Explain to patient that you are about to insert catheter
15) Insert catheter into tracheostomy until resistance is met
16) Suction patient for 10 to 15 seconds while you roll the catheter between your thumb and forefinger
17) Set artificial nose accurately after suctioning
18) Rinse catheter with water
19) Turn off suction machine and remove the catheter from the connecting tubing
20) Dispose of catheter while maintaining sterility of dominant hand
21) Doff PPE in the correct order (i.e. gloves, apron and mask)
22) Perform hand hygiene
23) Auscultate bronchial breath sounds
24) Explain that suctioning was finished 
Table 2. Questionnaire (5-point Likert scale). The questionnaire was composed of 12 items and divided into 5 categories.

\begin{tabular}{|c|c|}
\hline Category & Question \\
\hline Understanding & $\begin{array}{l}\text { 1) I could understand the skills (tracheostomy suctioning) well due to } \\
\text { first-person perspective } \\
\text { 2) I could understand the process of the skills well } \\
\text { 3) I could understand how to move the hands well }\end{array}$ \\
\hline Motivation & $\begin{array}{l}\text { 4) Viewing the VR-TM was interesting } \\
\text { 5) I think that the VR-TM enhances motivation to practice the skills } \\
\text { 6) I want to study other nursing skills using the VR-TM }\end{array}$ \\
\hline Immersion & $\begin{array}{l}\text { 7) I felt as if I was performing the skills } \\
\text { 8) I felt immersed in the virtual environment } \\
\text { 9) I could concentrate on viewing the VR-TM }\end{array}$ \\
\hline Control & $\begin{array}{l}\text { 10) I no longer want to experience the VR-TM } \\
\text { 11) The VR-TM is useless to understand the nursing skills }\end{array}$ \\
\hline Side effects & 12) I felt VR sickness (e.g. discomfort, headache or nausea) \\
\hline
\end{tabular}

VR-TM = virtual reality teaching material.

\subsection{Statistical Analysis}

All statistical analyses were performed using the $\mathrm{R}$ statistical software, version 3.2.3 (R Foundation for Statistical Computing). The level of probability for statistical significance was $\mathrm{p}<0.05$.

\section{Results}

\subsection{Questionnaire Rating}

Figure 3 shows the mean ratings of all question items. We tested whether positive/negative ratings were statistically higher/lower than zero (i.e. neutral) using a one-sample t-test. The ratings of Q1-9 (i.e. understanding, motivation and immersion) were significantly higher than zero (all, $\mathrm{t}_{35}>3.7 ; \mathrm{p}<0.001$ ), suggesting that participants affirmed these items. On the other hand, the ratings of Q10 and Q11 (i.e. control) were less than zero (both, $\mathrm{t}_{35}<-18.1$; $\mathrm{p}<0.001$ ), showing that participants denied these items. Although the rating of Q12 (side effect, mean $=-0.5)$ was significantly less than zero $\left(\mathrm{t}_{35}=-2.1 ; \mathrm{p}<0.05\right), 13$ participants $(36 \%)$ affirmed the item $(\geq 1)$ due to greater inter-subject variability $(\mathrm{SD}=1.4$; see an error bar $)$.

\subsection{Skill Assessment Test}

Table 3 shows mean scores of the skill assessment pretest and posttest for three groups. Figure 4 demonstrates score changes from the pretest to posttest (i.e. posttest minus pretest). With regard to the score changes, a one-way analysis of variance indicated a significant main effect $\left(\mathrm{F}_{2,33}=12.3, \mathrm{p}<0.001\right)$. Subsequent analysis using Tukey's honest significant difference test demonstrated that the score changes of the VR $(7.8 \pm 3.3$ points; mean \pm SD $)$ and TR groups $(6.0 \pm 2.6$ points) were significantly greater than the NO group $(2.1 \pm 2.6$ points) (both, $\mathrm{p}<$ $0.001)$. However, the difference between the VR and TR groups was not statistically significant $(\mathrm{p}=0.29)$. 
Table 3. Mean scores and standard deviation (SD) of the skill assessment pre- and post-test (range: 0 to 24 points) for each group. Values in parentheses indicate SD.

\begin{tabular}{cccc}
\hline & \multicolumn{3}{c}{ Group } \\
\cline { 2 - 4 } & NO group [n=12] & TR group [n $=12]$ & VR group [n=12] \\
\hline Pre-test & $12.8(3.8)$ & $14.1(3.9)$ & $13.5(4.1)$ \\
Post-test & $14.9(3.9)$ & $20.2(1.9)$ & $21.3(1.5)$ \\
\hline
\end{tabular}

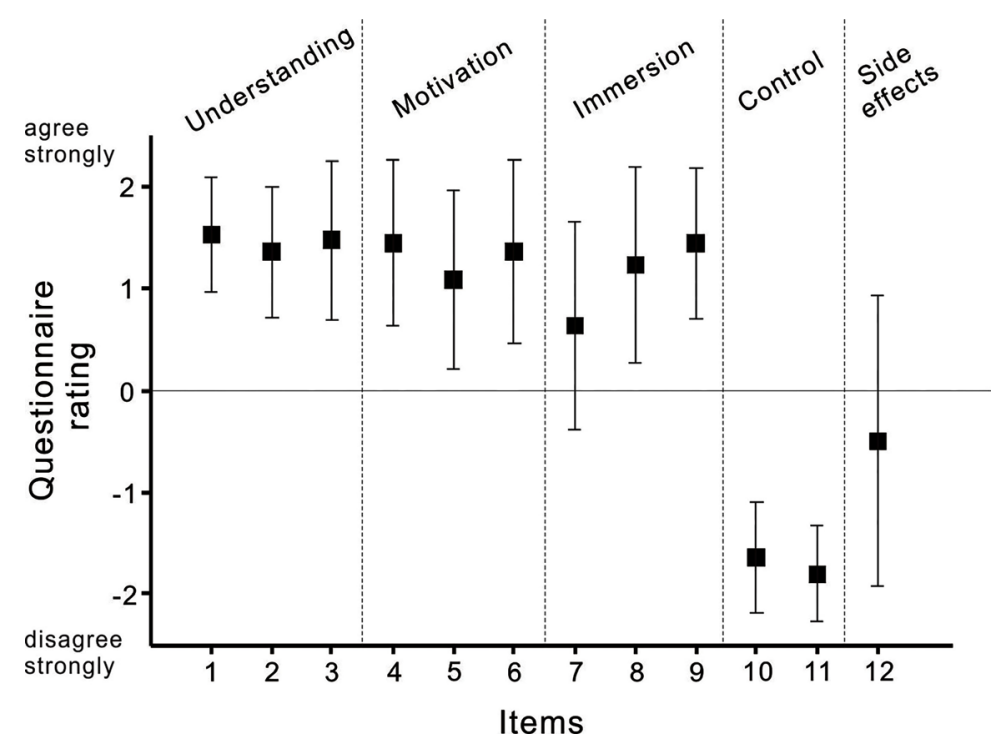

Figure 3. Mean questionnaire ratings and standard deviation (SD). Error bars represent $\pm 1.0 \mathrm{SD}$.

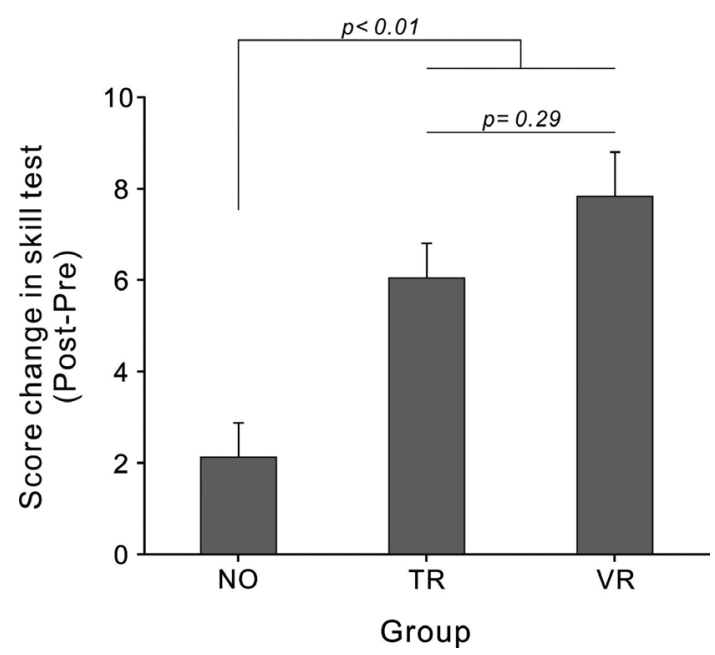

Figure 4. Mean score changes in the skill assessment tests (posttest minus pretest) of three groups. Error bars represent $+1.0 \mathrm{SE}$.

\section{Discussion}

In the questionnaire rating, the results from the target items (Q1-9) demonstrated that the participants evaluated the VR-TM usability favorably, a finding that was also supported by obvious denial of the control items (Q10-11: both, 
ratings $<-1.5)$. These results suggest that the combination of first-person video and the HMD may be promising as a teaching material for nursing skills.

All ratings for understanding (Q1-3) and motivation (Q4-6) were greater than +1 (i.e. affirmation). There would be at least two possible reasons for the high ratings regarding the understanding of the skill. First, when viewing demonstrator's hand actions from a third-person perspective, the learners have to mentally transform the representation into their own perspective. Conversely, such a mental process is not required when viewing the first-person video, resulting in easier understanding of the skill. Second, compared to the third-person video, the first-person video more prominently displayed the demonstrator's hands and arms (see Figure 1(b) and Figure 1(d)). This could give observers more detailed information about the demonstrator's hand actions.

Regarding high ratings of motivation, the result of Q4 suggests that the VR-TM would deliver interesting and exciting experiences for participants. Indeed, most of the participants experienced VR for the first time during the experiment. Thus, we speculated that the interest could be linked to feelings of immersion induced by the combination of the first-person video and the HMD. On the other hand, high motivation to practice the nursing skills (Q5 and Q6) could be partly related to the understanding of the skill. That is, the easily understandable VR-TM might lead to high motivation to practice the skill.

The high ratings for Q8 and Q9 suggest that the VR-TM with the HMD could offer participants feelings of immersion, which is an essential aspect of VR. In addition, the result of Q7 demonstrates that a large number of participants (86\%; [31/36]) felt as if they were performing the nursing skill while seeing the VR-TM (rating $>+1)$. Actually, previous studies in cognitive science reported that the first-person perspective facilitates visuomotor information processing and motor planning compared to the third-person perspective [6] [7]. Given these findings, it appears that observing the first-person video via the HMD enhanced the visuomotor processes in this study.

From the result for Q12 (i.e. side effects), it was found that approximately one-third of participants experienced mild VR sickness (e.g. discomfort, headache or nausea); though duration of the video was relatively short (six minutes). It is known that the majority of VR users experience VR sickness due to discrepancies between different sensory modalities (i.e. visual and vestibular information) [8]. In this study, motion (especially rotation) of the demonstrator's head with the wearable camera induced large camera shakes, which presumably caused VR sickness. Accordingly, we need to improve the VR-TM further by minimizing camera shakes and/or inserting a blank screen if the demonstrator's head moves extensively.

Regarding the skill assessment test, the VR and TR groups showed higher score changes than the NO group, suggesting clear intervention effects. However, no significant difference was identified between the two groups, though the mean score was slightly greater in the VR group compared to the TR group. 
While this result could be interpreted as less efficient, it might be due to the pilot study's methodological issues and study limitations. First, the participants had already learned the tracheostomy suctioning skills, so that the pretest scores ranged from eight $(33 \%)$ to 20 points $(83 \%)$ across groups (mean $=13.5$ points; $56 \%$ ). Consequently, ceiling effects might conceal the group differences in the posttest. Second, as the posttest was administered immediately after the intervention phase, we were unable to evaluate longer retention effects. Third, because the participants' demonstration was qualitatively assessed by the checklist in the skill assessment test, the present study did not evaluate changes to participants' demonstration time or time to decision-making (i.e. quantitative indices). Finally, the lack of differences between the VR and TR groups could be due to a lack of statistical power because the sample size (12 in each group) was relatively small. Given these issues, future studies should increase the number of participants, and examine long-term effects of the VR-TM, using both qualitative and quantitative evaluation indices.

\section{Conclusion}

The results of this pilot study suggest that the combination of first-person demonstration video and the HMD might be promising as a teaching material for nursing skills, though further improvements are necessary.

\section{Acknowledgements}

We would like to gratefully thank Mr. Kazuya Obara and Miss Kazue Uda for their help with data collection. This study was supported by Bunkyo Gakuin University.

\section{Authors' Contributions}

H. S. and S. S. contributed equally to this work.

\section{Conflicts of Interest}

The authors declare no conflicts of interest regarding the publication of this paper.

\section{References}

[1] Fukuta, J. and Morgan, J. (2018) First-Person Perspective Video to Enhance Simulation. The Clinical Teacher, 15, 231-235. https://doi.org/10.1111/tct.12659

[2] Davis, R.L. (2009) Exploring Possibilities: Virtual Reality in Nursing Research. Research and Theory for Nursing Practice, 23, 133-147. https://doi.org/10.1891/1541-6577.23.2.133

[3] Weiss, S., Bongartz, H., Boll, S. and Heuten, W. (2018) Applications of Immersive Virtual Reality in Nursing Education-A Review. Clusterkonferenz Zukunft der Pflege-Innovative Technologien für die Praxis, Oldenburg.

[4] Dyer, E., Swartzlander, B.J. and Gugliucci, M.R. (2018) Using Virtual Reality in Medical Education to Teach Empathy. Journal of the Medical Library Association, 
106, 498-500. https://doi.org/10.5195/JMLA.2018.518

[5] Butt, A.L., Kardong-Edgren, S. and Ellertson, A. (2018) Using Game-Based Virtual Reality with Haptics for Skill Acquisition. Clinical Simulation in Nursing, 16, 25-32. https://doi.org/10.1016/j.ecns.2017.09.010

[6] Vagt, S., Taylor, P. and Hopkins, B. (2003) Visuomotor Priming by Picture of Hand Postures: Perspective Matters. Neuropsychologia, 41, 941-951. https://doi.org/10.1016/S0028-3932(02)00319-6

[7] Kelly, R.L. and Wheaton, L.A. (2013) Differential Mechanisms of Action Understanding in Left and Right-Handed Subjects: The Role of Perspective and Handedness. Frontiers in Psychology, 4, 957. https://doi.org/10.3389/fpsyg.2013.00957

[8] Sharples, S., Cobb, S., Moody, A. and Wilson, J. (2007) Virtual Reality Induced Symptoms and Effects (VRISE): Comparison of Head Mounted Display (HMD), Desktop and Projection Display Systems. Displays, 29, 58-69.

https://doi.org/10.1016/j.displa.2007.09.005 